\title{
Yarn Hairiness Characterization Using Two Orthogonal Directions
}

\author{
Vítor H. Carvalho, Paulo J. Cardoso, Michael S. Belsley, Rosa M. Vasconcelos, and Filomena O. Soares
}

\begin{abstract}
We demonstrate that one can adequately characterize yarn hairiness by imaging the yarn along a single projection direction using coherent optical processing. A system that simultaneously characterizes the yarn hairiness along two orthogonal projection directions was constructed. Provided that a sufficiently high number of yarn segments are sampled, a strong statistical correlation is obtained between the results in each direction. The resulting images are generated using coherent optical signal processing with a Fourier high-pass spatial filter. This filter blocks the yarn core and produces a signal that highlights the sharp transitions in the transmission of the yarn. Essentially, only the small fibers responsible for the hairiness and the yarn core contours are present. Experimental results are presented for a $62-\mathrm{g} / \mathrm{km}$ yarn possessing a high degree of hairiness.
\end{abstract}

Index Terms-Electronic instrumentation, optical sensors, orthogonal directions, signal processing, yarn hairiness.

\section{INTRODUCTION TO YARN HAIRINESS ANALYSIS SYSTEMS}

$\mathbf{T}$ HE APPEARANCE of fabrics is greatly influenced by the level of hairiness of the yarn. Hairiness is the result of released fibers over the strand. Fig. 1 presents an example of hairiness [1]-[6].

The measurement of yarn hairiness allows one to quantify several statistical parameters that are important in characterizing the quality of yarns and, subsequently, fabrics.

Common methods of measuring hairiness are traditionally based on microscopic methods, weighing methods, and photoelectric methods [7], [8], whereas image processing methods are currently under development [8].

Microscopic methods consist of observing the yarn under a microscope to measure the hairiness. Several enterprises have used this methodology, such as Barella, which counts the number of protruding hairs, loops, and respective hair lengths in a specific yarn section; Pillay, which follows this method in a 10-mm length; Onions and Yates, which considers photo-

Manuscript received July 31, 2007; revised January 4, 2008. First published September 30, 2008; current version published February 9, 2009. This work was supported by the Portuguese Foundation for Science and Technology (FCT) under Scholarship BD/19028/2004. The Associate Editor coordinating the review process for this paper was Dr. Juha Kostamovaara.

V. H. Carvalho and F. O. Soares are with the Department of Industrial Electronics (DEI), Minho University, Campus de Azurém, 4800-058 Guimarães, Portugal (e-mail: vcarvalho@dei.uminho.pt; fsoares@dei.uminho.pt).

P. J. Cardoso and M. S. Belsley are with the Department of Physics (DF), Minho University, Campus de Gualtar, 4710-057 Braga, Portugal (e-mail: pjcardoso@fisica.uminho.pt; belsley@ fisica.uminho.pt).

R. M. Vasconcelos is with Department of Textile Engineering (DET), Minho University, Campus de Azurém, 4800-058 Guimarães, Portugal (e-mail: rosa@det.uminho.pt).

Color versions of one or more of the figures in this paper are available online at http://ieeexplore.ieee.org.

Digital Object Identifier 10.1109/TIM.2008.2005082

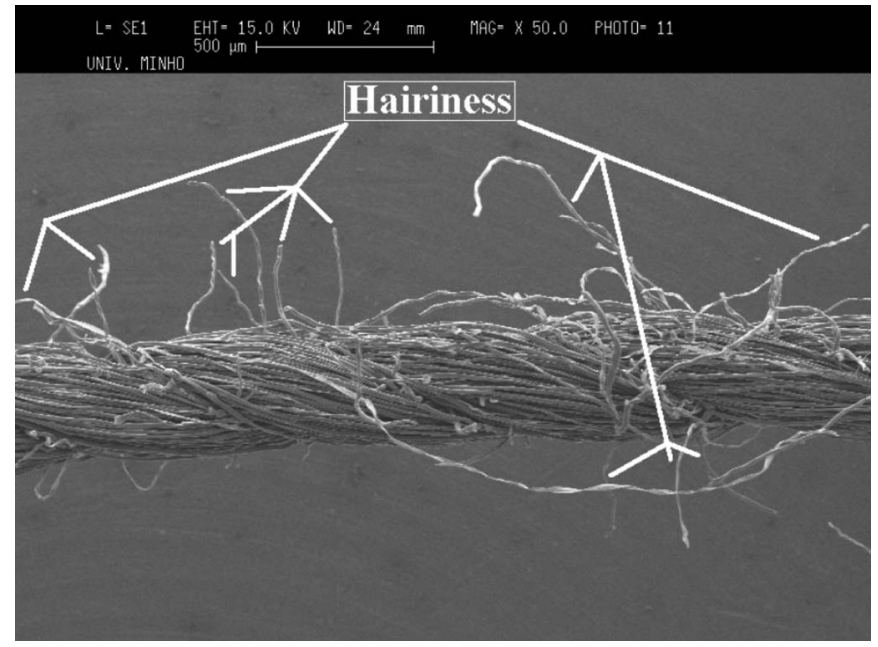

Fig. 1. Identification of yarn hairiness in an electron microscope photograph.

graphic standards to classify the hairiness in a projected yarn image; and Jedryka, which photographs the yarn and then defines the boundaries and equidistant lines by counting the number of hairs that intersect these lines. Nevertheless, in microscopic methods, it is difficult to identify the yarn boundaries because looped fibers, wild fibers, low twisted portions, and diameter variations greatly complicate the identification process. Reliable measurements require a high number of samples, which is time consuming.

Weighing techniques are based on the difference of yarn mass before and after singeing (burning the protruding hairs). However, a high number of samples should be analyzed for a clear estimate. Furthermore, singeing often does not completely remove the hairiness of a yarn, particularly with shorter lengths. Thus, this methodology lacks precision.

To overcome these problems, some equipment employs photoelectric methods. Shirley (Atlas Hairiness Tester) uses a lightemitting diode beam/photocell pair (measuring head adjustable between 1 and $10 \mathrm{~mm}$ ) to count the number of interruptions that the protruding hairs provoke in the light beam. Zewigle (Hairiness Tester) also uses the same methodology but defines nine length zones between 1 and 12 or $15 \mathrm{~mm}$ using different optical channels. Uster Tester and Premier Electronic Tester use homogeneous rays of parallel light from an infrared light source, where the scattered light from the protruding hairs of yarn is detected by an optical sensor and then converted into an electronic signal. In this approach, which is essentially dark field microscopy, the body of the yarn is dark, and the protruding fibers are bright.

Despite of the advantages of the photoelectric methods in comparison with the weighting and microscopic approaches, these have also several drawbacks. The systems based on 
photocells have difficulty in correctly characterizing the length of hairs as they can take on a variety of geometrical shapes. The commercial equipment, which uses infrared light, significantly improved the previous methods. However, it employs crossed optical polarizers to extract the yarn core, which easily can remove important signal components as the hairiness orientation can alter the polarization of the scattered light. Moreover, the contours of the yarn can also be improperly considered a signal due to hairs.

In addition, image processing methods are still under development, but for a correct classification of hairiness, they require a well-defined algorithm to identify the hairs from the main core, a camera with a high level of optical magnification, and a computer-based system with significant computational resources to process results in useful time, all of which are characteristics that severely increase the cost.

As an alternative to the aforementioned techniques, we have developed a coherent optical signal processing technique based on Fourier analysis to characterize yarn hairiness using a singular projection [1]-[6]. This system overcomes the referred drawbacks of other methods because it uses a signal reference for $0 \%$ of yarn hairiness [5], it does not consider the yarn contours, and the optical configuration is not dependent on the hairiness orientation. Moreover, the obtained signal is proportional to the length of hairs. These characteristics are fundamental for a reliable and precise characterization of hairiness. However, a full hairiness classification should require several different and simultaneous projections. However, we have verified that as hairiness is randomly distributed over the yarn [2], there is a high probability, considering the length or yarn analyzed in each test, that on average, the amount of hairiness is uniformly distributed over a full rotation of the yarn. With this study, we verify that the results obtained using two orthogonal projections give nearly identical results. As a consequence, a single projection is sufficient for the correct determination of the full amount of hairiness in yarn.

\section{DeVeloped Yarn Hairiness System CHARACTERIZATION}

The method we have developed to characterize hairiness along a single direction consists of a single-mode visible diode laser as a light emitter and a photodiode as a receiver, plus an optical setup to analyze yarn hairiness using a coherent optical signal processing technique based on Fourier optics (1) [4]. Consider a coherent plane wave that illuminates an object and then propagates through a lens toward its principle focal plane on the far side. Fourier optics implies that the electric field distribution in the focal plane of the lens is the Fourier transform of the field distribution in the object plane [9]. The lower spatial frequencies of the image will be concentrated close to the optical axis, whereas the higher spatial frequencies will be located further from the optical axis. Mathematically, the field distribution in the principal focal plane of the lens will be given by the following expression:

$$
F\{g\}=\iint_{-\infty}^{\infty} g(x, y) e^{-j 2 \pi\left(V_{x} x+V_{y} y\right)} d x d y
$$

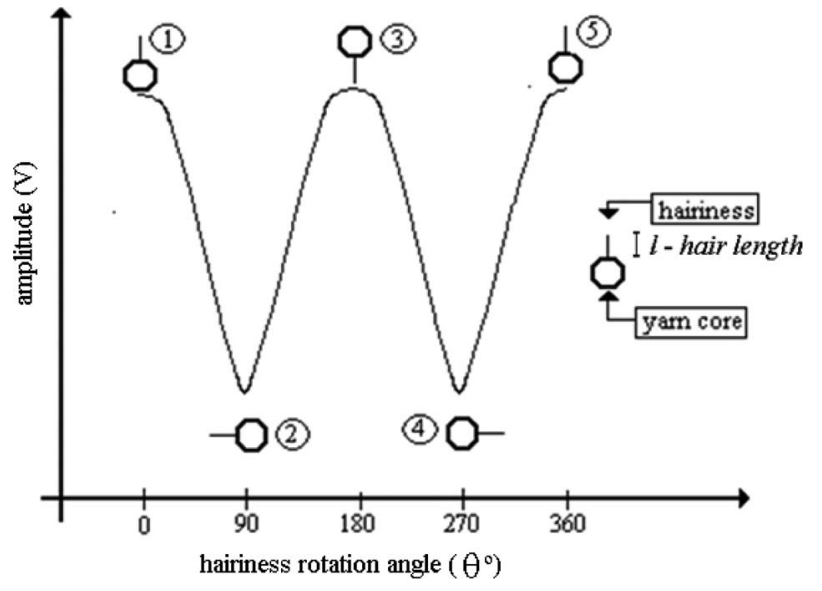

Fig. 2. Example of the absolute cosine signal resulting from a $360^{\circ}$ projection of one straight hair of length $l$ using a single photodiode.

where $g(x, y)$ describes the field distribution in the object plane, and $V_{x}$ and $V_{y}$ are the respective spatial frequencies (in cycles per meter) along the $x$ - and $y$-directions. The characteristic spatial frequency associated with an object of size $d$ is given by

$$
V \approx \frac{\lambda \cdot f}{d}
$$

where $V$ is the spatial frequency (in cycles per meter), $\lambda$ is the wavelength (in meters), and $f$ is the focal distance (in meters) of the Fourier lens.

Sharp transitions from light to dark in the object plane will result in high spatial frequencies, whereas the nearly uniform background illumination possesses only very low spatial frequencies. By placing a small round opaque filter centered on the optical axis in the Fourier plane, the low-frequency background information is blocked, whereas the information about the sharp transitions from light to dark that occur at the yarn boundaries and individual fibers (hairiness) can pass through. When imaged by a second lens, this high-pass filter setup results in dark and bright images, where the bright signal contains information about yarn hairiness that is converted into a proportional current intensity by a photodiode [4].

Considering a single coherent imaging system, if yarn containing a single straight fiber hair of length $l$ thrusting out from the core is rotated over $360^{\circ}$, an absolute cosine signal with an amplitude of the length of the hairiness is obtained (Fig. 2). Essentially, the system would record a signal proportional to the cosine of the angle between the direction of the hair fiber and the plane perpendicular to the incident light's direction. Maximum projections are obtained at orientations referenced by 1,3 , and 5 in Fig. 1 . In contrast, a minimum signal would be obtained for orientations referenced by 2 and 4 when the hair is aligned along the incident light's propagation direction.

If a second coherent imaging system is constructed along a direction orthogonal to the first system (Fig. 3), a complementary signal (absolute sine) is obtained (Fig. 4). The effective length of the protruding fiber $l$ is, in this case, proportional to the absolute sine of the angle between the hair's direction and the plane perpendicular to the incident direction of the initial light beam. 


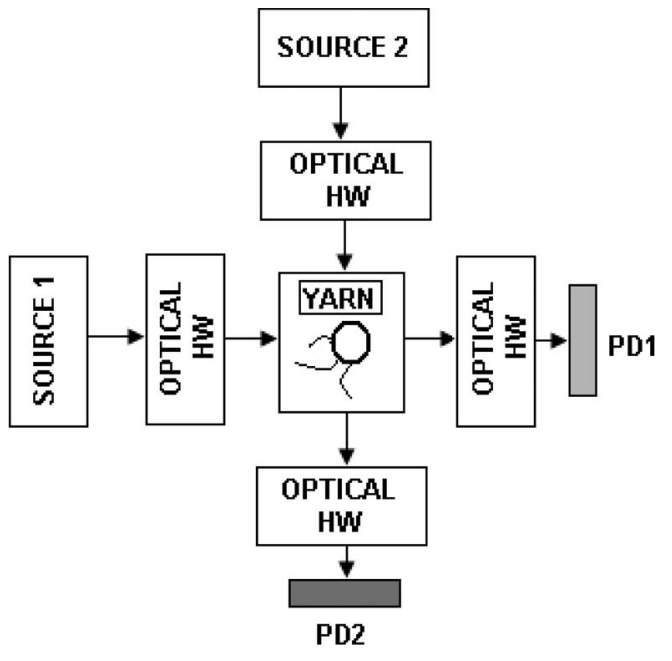

Fig. 3. Configuration of a system to quantify yarn hairiness using two orthogonal directions.

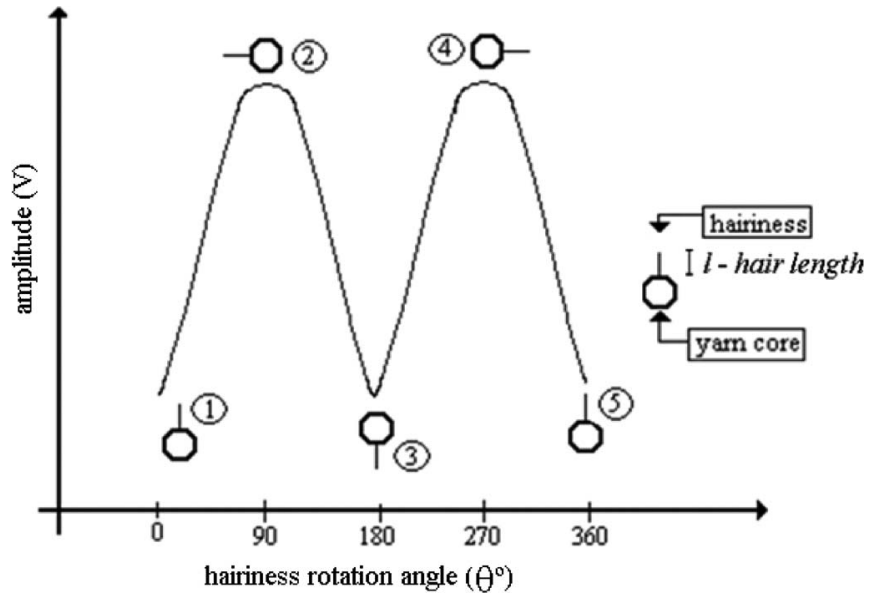

Fig. 4. Example of the absolute sine signal resulting from a $360^{\circ}$ projection of one straight hair of length $l$ for a second photodiode orthogonally positioned $\left(90^{\circ}\right)$ relatively to the first photodiode.

Furthermore, if several hairs are positioned over the yarn with different angular displacements, the two systems register signals corresponding to the net projections of the illuminated hairs in the two planes perpendicular to the incident light's directions.

Mathematically, for an arbitrary direction of a given hair segment, the signals $S_{1}$ and $S_{2}$ recorded by the two photodiodes (PD1 and PD2, respectively) will be proportional to the absolute cosine and the absolute sine of the hair's direction relative to the detection plane of the first photodiode, i.e.,

$$
S_{1} \sim l|\cos (\theta)| \quad S_{2} \sim l|\sin (\theta)| .
$$

For an arbitrary hair orientation, the signal acquired by a single photodiode corresponds to the following average:

$$
S_{1} \sim l \frac{2}{\pi} \int_{0}^{\pi / 2} \cos (\theta) d \theta=\frac{2 l}{\pi}[\sin (\theta)]_{0}^{\pi / 2}=\frac{2 l}{\pi} \approx 0.64 l .
$$

This signal is calculated between 0 and $90^{\circ}(\pi / 2)$, as in this angular range, the full-amplitude variations of the sampled signal are then repeated over the following $270^{\circ}$.

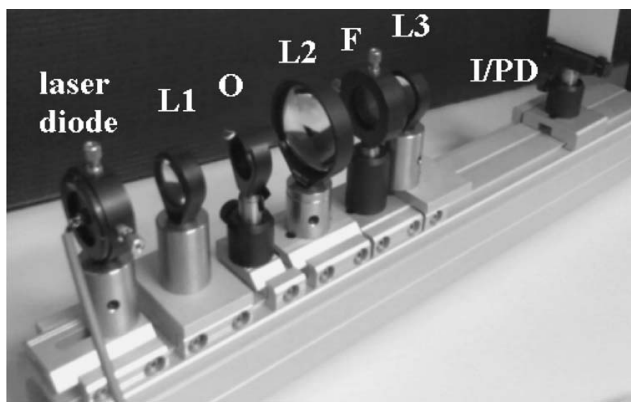

Fig. 5. Custom-developed optical yarn measurement HW for a single direction.

A similar calculation also holds for the hairiness detected by the photodiode in the orthogonal direction. On average, assuming a random distribution of protruding hairs, the total amount of hairiness $S_{t}$ will be $\pi / 2(1 / 0.64)$ times larger than that detected along a single projection, i.e.,

$$
S_{t}=\frac{\pi}{2} S_{1} \approx 1.57 S_{1}
$$

Provided the distribution of protruding hairs is random in direction and the area of "intersecting hair projections" is negligible, the aforementioned relation should always prove valid. To explore the validity of this claim for an actual yarn with a high degree of hairiness, a double coherent imaging setup was constructed to simultaneously characterize yarn hairiness along two orthogonal directions.

\section{EXPERIMENTAL CONFIGURATION}

To simultaneously analyze yarn hairiness along two orthogonal projection directions, we have duplicated the electronic and optical hardware (HW) used in the single-direction hairiness analysis system referred in the previous section. Fig. 5 presents the optical HW used for a single-direction yarn hairiness measurement system [1]-[6].

We used a coherent optical imaging technique to obtain an optical signal proportional to the amount of hairiness present on the yarn being sampled. The main components of our optical system are a collimated coherent illumination source, a high numerical aperture lens used to form the Fourier transform image of the yarn under study, a custom-made high-pass spatial filter [9]-[12], and a second lens to create a final image in the detector plane (position of the photodetector in Fig. 5, i.e., I/PD).

For the illumination source, we use a diode laser (Eudyna FLD6A2TK) that emits light at $685 \pm 10 \mathrm{~nm}$ in both a single transverse and a single longitudinal mode with a particularly low aspect ratio of 1.3. Emitting in a single longitudinal mode, the coherence length of the laser light is expected to be greater than several tens of meters. The light from this laser was collimated using a single planoconvex lens of $40 \mathrm{~mm}$ focal length (L1). After illuminating the sample under study (O), the light passes through a $60-\mathrm{mm}$ focal length, $50-\mathrm{mm}$-diameter planoconvex lens (L2) used to obtain a spatial Fourier transform of the object in its principal focal plane. A roughly 1-mmdiameter opaque target $(\mathrm{F})$ placed in the Fourier plane blocks all spatial frequencies below $10 \mathrm{~mm}^{-1}$, corresponding to a characteristic size of $100 \mu \mathrm{m}$ (or larger) in the object plane. 


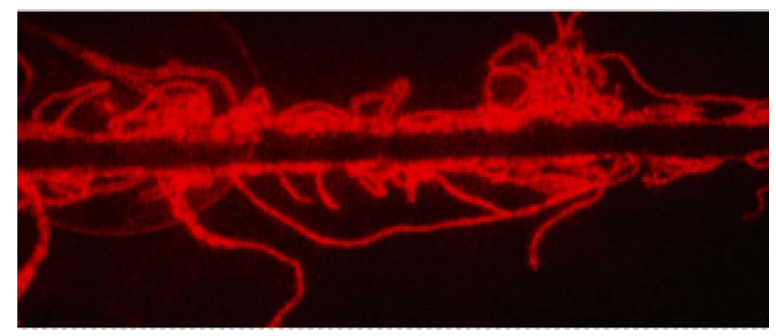

Fig. 6. Example of an image resulting from coherent optical signal processing using a high-pass spatial filter.

Fortunately, there is a good separation in the characteristic length scales of the yarns. Textile yarns typically have diameters ranging from a few hundreds of a few micrometers up to millimeters, whereas the small hairs protruding from the yarn are single fibers with diameters typically less than $10 \mu \mathrm{m}$. The filter is chosen to pass the information with regard to the small fibers while eliminating almost entirely the nearly constant background light due to the laser light that is not incident on the sample. However, the amount of light transmitted by the spatial filter is relatively small, i.e., roughly three to four orders of magnitude less than the full power emitted by the laser. A third planoconvex lens of $60 \mathrm{~mm}$ focal length (L3) is used to form a final filtered image, which highlights the sharp transitions in the object, i.e., basically the core boundary of the fiber and any small protruding hairs from the yarn core (see Fig. 6). In this main setup, all lenses have a broadband antireflective coating. In addition, an interference filter centered at $680 \mathrm{~nm}$ with a bandpass (full-width at half-maximum) of $10 \mathrm{~nm}$ and a peak transmission of 50\% (Thorlabs FB680-10) can directly be placed in front of the detector to limit the amount of stray ambient light detected.

Fig. 7 presents the electronic instrumentation HW developed for one direction, which is characterized by a bandwidth of $1 \mathrm{MHz}$, an offset voltage of $\pm 10 \mu \mathrm{V}$, a noise level inferior to $10 \mathrm{mV}$, and a noise-equivalent power of $3.1 \times 10^{-15} \mathrm{~W} / \mathrm{Hz}^{1 / 2}$. The output power of the lasers during measurements has variations inferior to $1 \%$. The goal of the electronic $\mathrm{HW}$ is to obtain a voltage proportional to the brightness of the final image [4]. The output of a transimpedance amplifier [13], which converts the current generated by the photodiode into a voltage, is read by the USB-6251 data-acquisition (DAQ) board, from National Instruments. Some of the main features of the USB-6251 DAQ board are as follows: 16 bits of resolution, a maximum acquisition rate of $1.25 \mathrm{MS} / \mathrm{s}$, a maximum range of $\pm 10 \mathrm{~V}$, and a \pm 1 least significant bit.

To build the optical HW to characterize the hairiness along the orthogonal projection, we have used the same type of optical elements. However, due to equipment limitations, the elements in the second optical arm have slightly different characteristics; the lenses used have slightly different focal lengths, and the laser sources operate at different wavelengths and powers. Nonetheless, the operation principal is identical, whereas the associated electronic HW is exactly reproduced. Moreover, to ensure that the beam area incident on photodiode is precisely the same, we built two 5-mm-high similar windows (final beam area of $5 \mathrm{~mm} \times 10 \mathrm{~mm}$ ) and placed them over the active area of the photodiodes. To obtain approximately the same output signal amplitude in both setups, the gains used in the transimpedance amplifiers need to be differently adjusted for each setup to compensate for the slight differences in the optical HW and light sources (source 1: helium-neon (HeNe) laser with a wavelength of $632.8 \mathrm{~nm}$, with a polarization contrast of more than 100:1; source 2: laser diode with a wavelength of approximately $685 \mathrm{~nm}$, as previously referred, with a polarization contrast of better than $100: 1)$. Fig. 8 presents a schematic of the overall optical HW setup for two directions.

In the several experimental runs performed, the results showed, as expected, that the majority of the signal detected by photodiode 1 was due to the light originating from laser source 1 . However, a small component of the light from laser source 2 is also scattered and detected by photodiode 1 . This indirect or cross signal was greatly reduced, in the case of photodiode 2 , by placing a narrow-band interference filter in front of the photodiode, which rejects the vast majority of the light that propagates to photodiode 2 from laser source 1 (see Fig. 8). A small amount of light from laser source 1 is nonetheless transmitted since the interference filter has a limited acceptance angle. To take into account the small cross signals, we repeated each experiment using three different scenarios.

a) Laser 1 on-laser 2 off. The signal in photodiode 1 is directly transmitted, and the signal registered by photodiode 2 is due to the light scattered from laser source 1.

b) Laser 1 off-laser 2 on. The signal observed using photodiode 2 is the directed transmitted signal, whereas the signal detected by photodiode 1 is due to the light scattered from laser source 2 .

c) Laser 1 on-laser 2 on. Both photodiodes registered the directly transmitted signal from the respective source together with a scattered signal from the other laser source.

\section{EXPERIMENTAL RESULTS}

A $62-\mathrm{g} / \mathrm{km}$ strand of yarn with a high level of hairiness was tracked over 4800 samples of $5 \mathrm{~mm}$ each, corresponding to a total of $24 \mathrm{~m}$ of yarn, for each tested laser configuration.

In each arm, a small constant background signal exists, mainly due to the quality of the optical elements used in the setup. This background can easily be measured by acquiring signals without the presence of yarn. The signals without yarn are $0.134 \mathrm{~V}$ for channel 1 (photodiode 1) and $0.014 \mathrm{~V}$ for channel 2 (photodiode 2). There is no reflection if no yarn is placed in the object plane. Thus, these values only refer to the signal transmission of the direct laser beam for each photodiode and could be used for the three tested situations, as presented in Table I.

We have subtracted the voltage-without-yarn values from the acquisition signals for each situation described in Table I. Figs. 9 and 10 present the acquisition signals with the yarn placed in the object plane of channels 1 and 2, respectively, considering situation (a), i.e., laser 1 on-laser 2 off.

The signal obtained in channel 1 is, as expected, higher than that of channel 2 since this is the channel that receives the directly transmitted light. In addition, channel 2 contains an optical filter that severely reduces the light from laser source 1 scattered into its direction, causing a signal that 


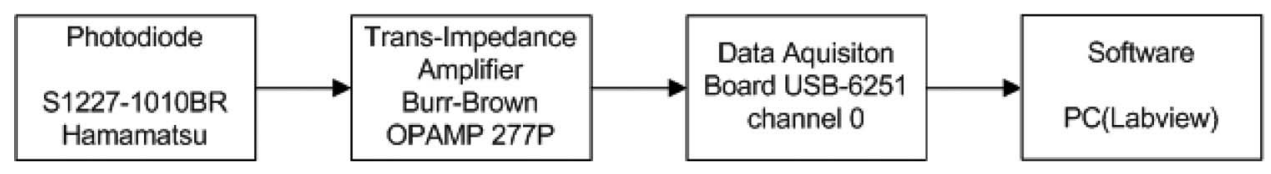

Fig. 7. Custom-developed electronic yarn measurement HW for a single direction.

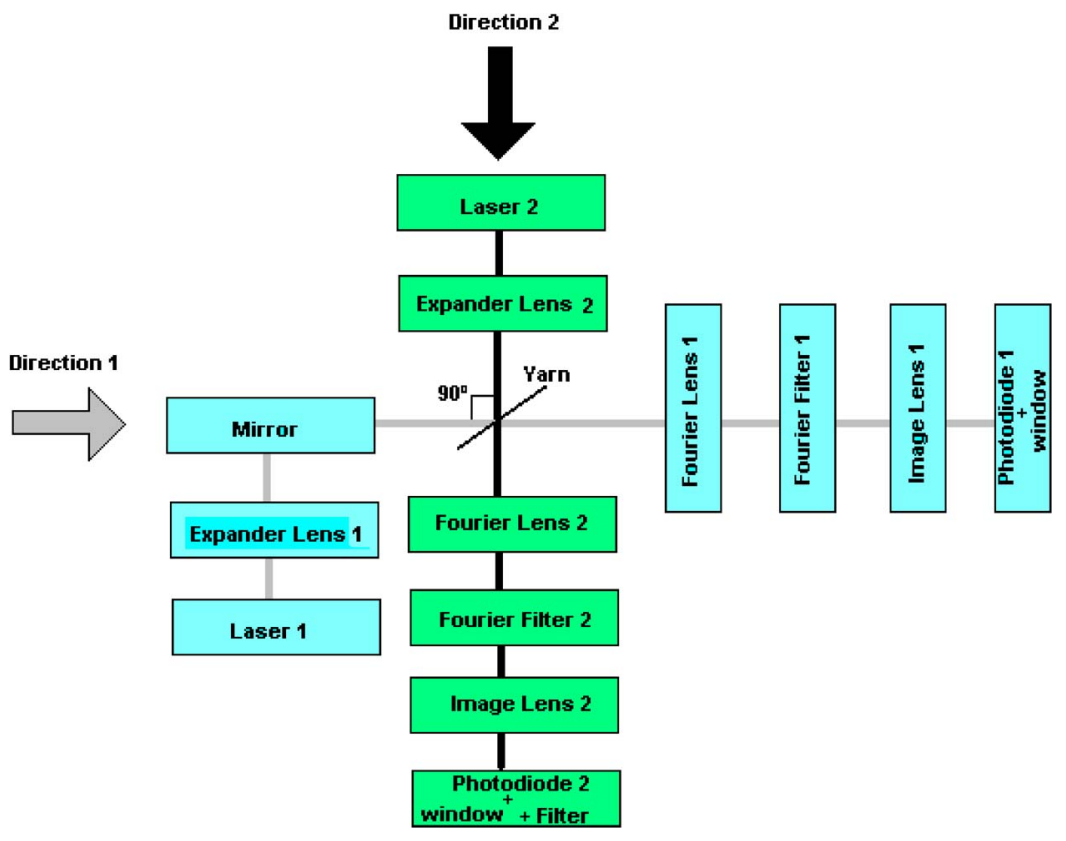

Fig. 8. Experimental configuration of an orthogonal yarn hairiness analysis.

TABLE I

VolTAGES Without YARN FOR EACH TESTED SitUATION

\begin{tabular}{|c|c|c|}
\hline Situation & $\begin{array}{c}\text { Voltage Without } \\
\text { Yarn channel 1 }\end{array}$ & $\begin{array}{c}\text { Voltage Without } \\
\text { Yarn channel 2 }\end{array}$ \\
\hline $\begin{array}{l}\text { Laser 1 on } \\
\text { Laser 2 off }\end{array}$ & $0.134 \mathrm{~V}$ & $0 \mathrm{~V}$ \\
\hline $\begin{array}{c}\text { Laser 1 off } \\
\text { Laser 2 on }\end{array}$ & $0 \mathrm{~V}$ & $0.014 \mathrm{~V}$ \\
\hline $\begin{array}{l}\text { Laser 1 on } \\
\text { Laser 2 on }\end{array}$ & $0.134 \mathrm{~V}$ & $0.014 \mathrm{~V}$ \\
\hline
\end{tabular}

Channel 1 (laser 1 on - laser 2 off)

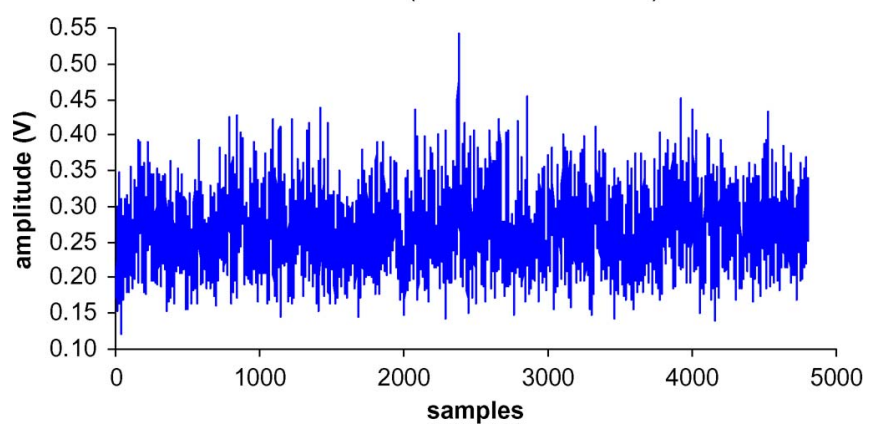

Fig. 9. Acquisition signal for channel 1 considering situation (a), i.e., laser 1 on-laser 2 off.

seems to be simply background, with an amplitude in the range $0.098-0.102 \mathrm{~V}$. In this case, the quantization observed is caused by the resolution of the analog-to-digital conversions performed by the DAQ board.

Moreover, to correctly classify the level of hairiness, we have determined the reference values ( $0 \%$ of hairiness) using

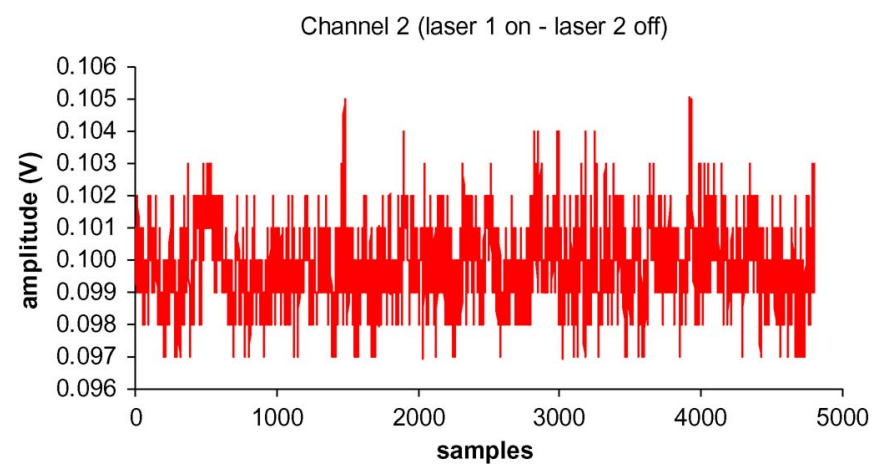

Fig. 10. Acquisition signal for channel 2 considering situation (a), i.e., laser 1 on-laser 2 off.

TABLE II

Statistical Reference, MeAn, AND SD for Figs. 9 AND 10

\begin{tabular}{|l|l|l|l|}
\cline { 2 - 4 } \multicolumn{1}{c|}{} & Reference & \multicolumn{1}{c|}{ Mean } & SD \\
\hline Channel 1 & $0.23 \mathrm{~V}$ & $0.27 \mathrm{~V}$ & $0.051 \mathrm{~V}$ \\
\hline Channel 2 & $0.098 \mathrm{~V}$ & $0.10 \mathrm{~V}$ & $0.001 \mathrm{~V}$ \\
\hline
\end{tabular}

a previously developed statistical method [5]. Table II presents the values obtained for the reference, average, and standard deviation (SD) of the signals displayed in Figs. 9 and 10.

In the following, we will use the average result obtained for channel 1 in Table II as a measure of the influence of laser 1 in channel 1 when both sources are connected. Channel 1 has a markedly larger SD value than channel 2 . While the SD of channel 1 should well reflect the degree of hairiness of the samples tested, the fluctuations in channel 2 could be associated with speckle due to the light scattered from the yarn core. 


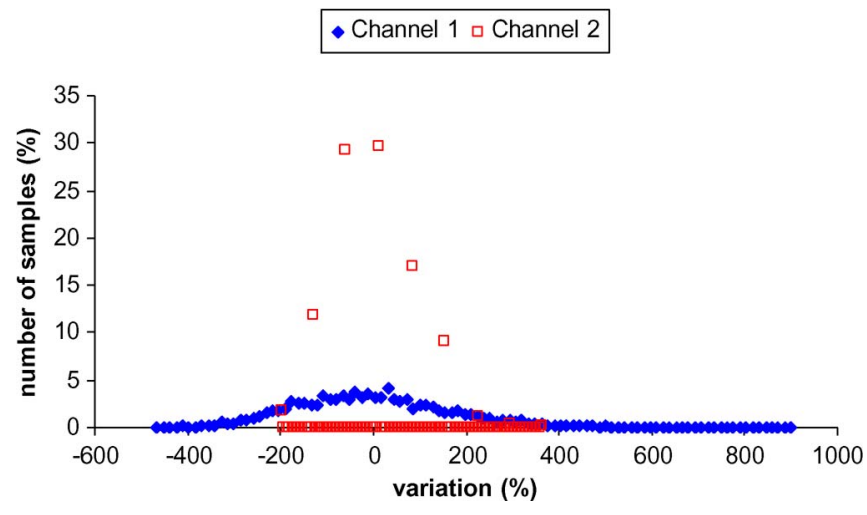

Fig. 11. Frequency diagrams of channels 1 and 2 for situation (a), i.e., laser 1 on-laser 2 off.

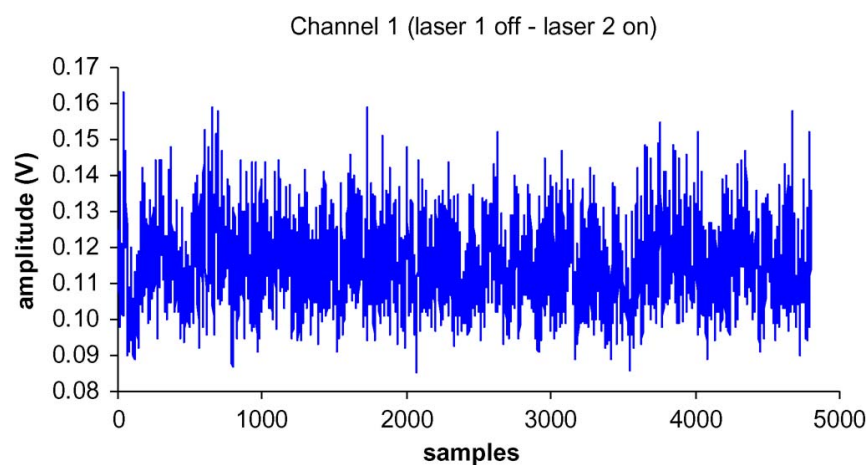

Fig. 12. Acquisition signal for channel 1 considering situation (b), i.e., laser 1 off-laser 2 on.

Subsequently, we have correlated both signals, considering the hairiness percentage variation over the mean value, using a frequency hairiness variation diagram, as presented in Fig. 11. The frequency diagram shows the acquisition sample distribution (i.e., percentage of the number of samples) for each channel, considering the mean value $(0 \%$ of variation) as a reference for a range variation divided into 100 intervals. This analysis will also be performed for scenarios (b) and (c).

Fig. 11 supports the idea that the signal from channel 2 is not well correlated with the hairiness measured by channel 1 and is probably associated with the laser light reflected off the yarn core. Considering the hairiness reference value, the percentage variation over the average SD for Figs. 9 and 10 also emphasizes this conclusion as they are quite different $(168.05 \%$ for channel 1 and $89.43 \%$ for channel 2).

Afterward, the previous analysis presented for situation (a) was performed for situation (b). Figs. 12 and 13 present the acquisition signals with the yarn placed in the object plane of channels 1 and 2, respectively, considering situation (b), i.e., laser 1 off-laser 2 on.

Again, the channel that measures the directly transmitted light has a much higher signal level. The reference $(0 \%$ of hairiness), average, and SD values were also determined for this situation. Table III presents the results of the reference, average, and SD values of Figs. 12 and 13.

As can be seen in Table III, the SD results from channel 2 are greater than those obtained from channel 1 , due to the direct transmission of the laser source to channel 2. Nevertheless,

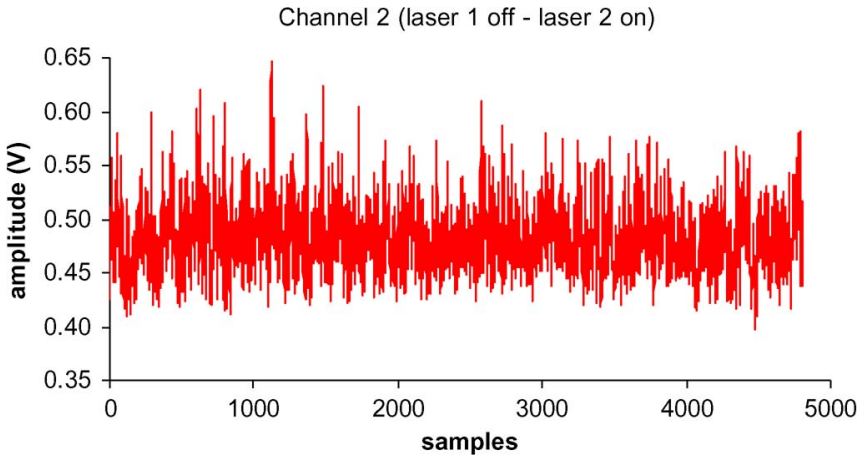

Fig. 13. Acquisition signal for channel 2 considering situation (b), i.e., laser 1 off-laser 2 on.

TABLE III

Statistical Reference, Mean, And SD for Figs. 12 And 13

\begin{tabular}{|l|l|l|c|}
\cline { 2 - 4 } \multicolumn{1}{c|}{} & Reference & Mean & SD \\
\hline Channel 1 & $0.11 \mathrm{~V}$ & $0.12 \mathrm{~V}$ & $0.012 \mathrm{~V}$ \\
\hline Channel 2 & $0.46 \mathrm{~V}$ & $0.48 \mathrm{~V}$ & $0.031 \mathrm{~V}$ \\
\hline
\end{tabular}

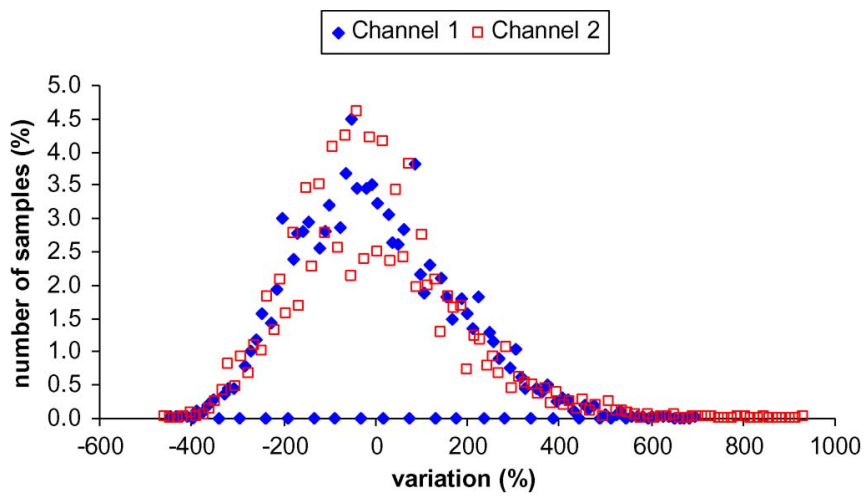

Fig. 14. Frequency diagrams of channels 1 and 2 for situation (b), i.e., laser 1 off-laser 2 on.

channel 1 receives some light scattered by the yarn since no filter is placed in it. A minor deviation of the SD results for both channels is obtained in comparison to situation (a). When both sources are connected, the average result obtained for channel 2 will be used to determine the influence of laser 2 in channel 2 . As in situation (a), we have correlated both signals, considering the hairiness percentage variation over the mean, using a frequency hairiness variation diagram, as presented in Fig. 14.

Observing Fig. 14, we can confirm that there is a high correlation for most of the tested intervals between the signals from channels 1 and 2. The absence of an optical interference filter results in a scattered signal that appears to be a fair measure of the yarn hairiness. The signals are, as expected, statistically close but significantly differ in amplitude, as the scattered signal is weaker than the direct signal. Considering the hairiness reference value, the percentage variation over the average SD for Figs. 12 and 13 also confirmed this conclusion as they are similar (170.65\% for channel 1 and $174.44 \%$ for channel 2).

Finally, in situation (c), we have determined the absolute signal variation caused by the nondirect sources using the following equation:

$$
\text { Vinds }=\text { Avbs }- \text { Avds }
$$


TABLE IV

INFLUENCE OF THE NONDIRECT SOURCES

\begin{tabular}{|c|c|c|c|}
\cline { 2 - 4 } \multicolumn{1}{c|}{} & \multicolumn{1}{c|}{$\begin{array}{c}\text { Mean result } \\
\text { direct } \\
\text { source only }\end{array}$} & $\begin{array}{c}\text { Mean result } \\
\text { both } \\
\text { sources }\end{array}$ & $\begin{array}{c}\text { Mean of } \\
\text { non direct } \\
\text { source }\end{array}$ \\
\hline Channel 1 & $0.27 \mathrm{~V}$ & $0.41 \mathrm{~V}$ & $0.14 \mathrm{~V}$ \\
\hline Channel 2 & $0.48 \mathrm{~V}$ & $0.49 \mathrm{~V}$ & $0.01 \mathrm{~V}$ \\
\hline
\end{tabular}

Channel 1 (laser 1 on - laser 2 on)

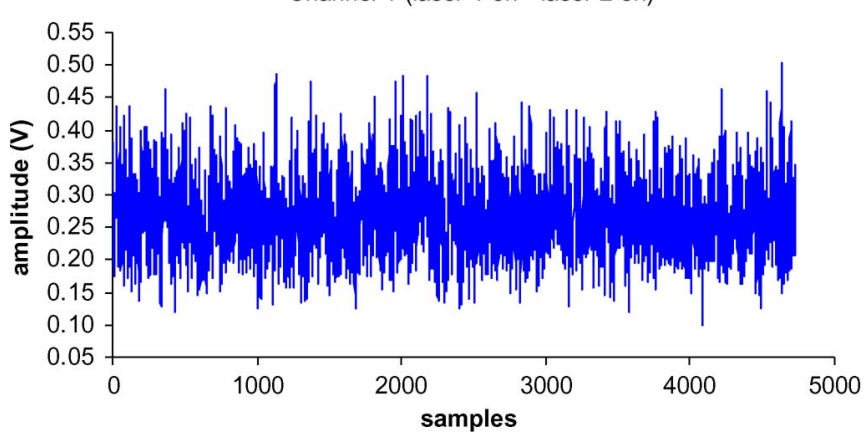

Fig. 15. Acquisition signal for channel 1 considering situation (c), i.e., laser 1 on-laser 2 on.

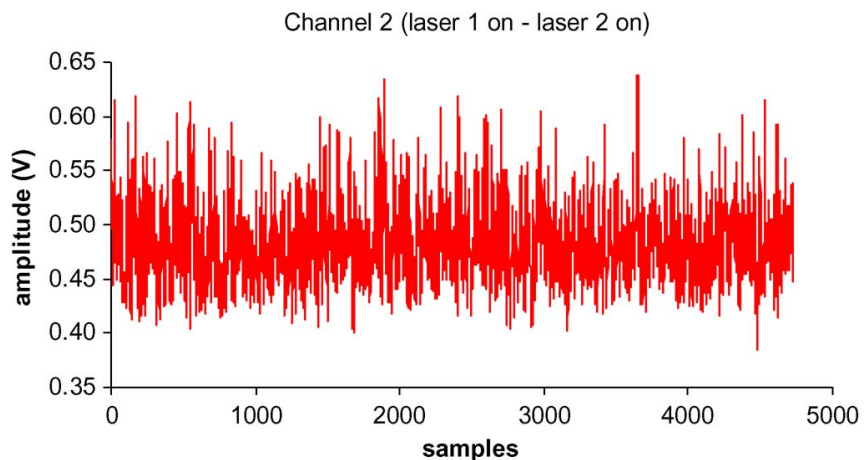

Fig. 16. Acquisition signal for channel 2 considering situation (c), i.e., laser 1 on-laser 2 on.

where Vinds is the voltage influence of the nondirect source (in volts), Avbs is the average voltage of the channel considering both sources are connected (in volts), and Avds is the average voltage of the channel considering only its direct source (in volts). These average values were found to be statistically constant for different sections of the same yarn (Table IV).

As channel 2 has a filter, we can verify from Table IV that there is a greater influence of the nondirect source on channel 1 $(0.14 \mathrm{~V})$ than on channel $2(0.01 \mathrm{~V})$. If no filter was placed in channel 2, the influence of the nondirect source on channel 2 should be closer to the average value of channel 2 in Table III $(0.10 \mathrm{~V})$. However, as in the applied case, the filter has also a direct source; thus, the background noise pattern observed in Fig. 10 is severely reduced, blocking almost the entire undesired signal.

The next step consisted of subtracting the influence of the nondirect source in each acquisition signal considering both sources. Figs. 15 and 16 present the acquisition signals with the yarn placed in the object plane of channels 1 and 2, respectively, considering situation (c), i.e., laser 1 on-laser 2 on.

Analyzing Figs. 15 and 16, it is possible to verify that due to their higher amplitudes, the signals of channels 1 and 2 are
TABLE V

Statistical Reference, Mean, AND SD fOR Figs. 15 AND 16

\begin{tabular}{|l|l|l|c|}
\cline { 2 - 4 } \multicolumn{1}{c|}{} & Reference & \multicolumn{1}{c|}{ Mean } & SD \\
\hline Channel 1 & $0.23 \mathrm{~V}$ & $0.27 \mathrm{~V}$ & $0.058 \mathrm{~V}$ \\
\hline Channel 2 & $0.46 \mathrm{~V}$ & $0.48 \mathrm{~V}$ & $0.035 \mathrm{~V}$ \\
\hline
\end{tabular}

\section{- Channel 1 口 Channel 2}

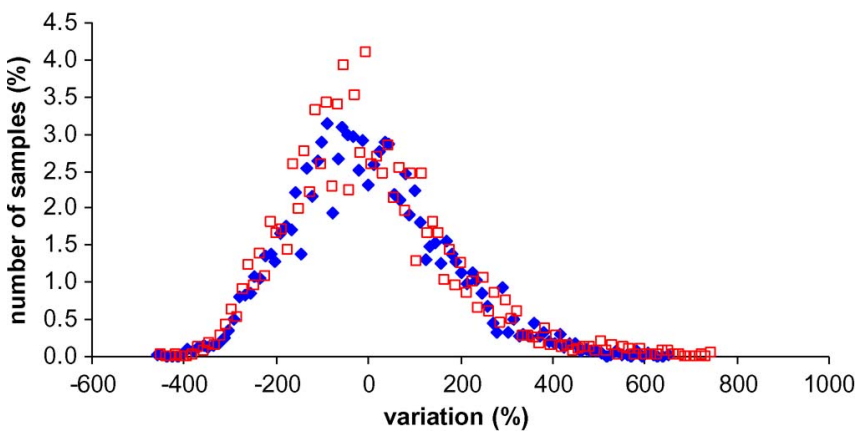

Fig. 17. Frequency diagrams of channels 1 and 2 for situation (c), i.e., laser 1 on-laser 2 on.

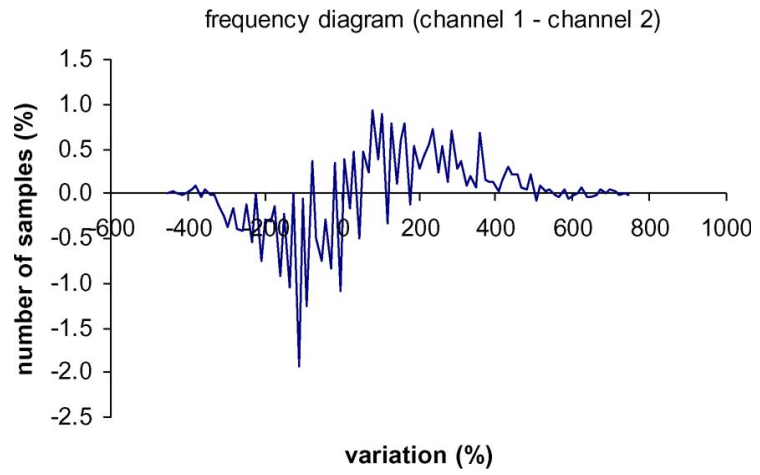

Fig. 18. Amplitude differences in each interval of Fig. 17.

directly transmitted. The differences verified in amplitude are related to the different source powers and slightly different gains of the amplifiers.

Table $\mathrm{V}$ presents the results of the reference $(0 \%$ of hairiness), average, and SD values of Figs. 15 and 16.

As expected, looking at Table $\mathrm{V}$, the reference and mean values are equal, whereas the SD values are very close to those obtained for situations (a) and (b), considering the direct source for each specific channel.

Then, using a frequency hairiness variation diagram, as presented in Fig. 17, we have correlated both signals, considering the hairiness percentage variation over the average.

Analyzing Fig. 17, we verify that both signals are highly correlated with regard to the number of samples obtained for each tested interval. Considering the hairiness reference value, the percentage variation over the average SD for Figs. 15 and 16 also confirmed this conclusion as they are in close agreement (162.83\% for channel 1 and $168.06 \%$ for channel 2). The differences of amplitudes in the number of samples (in percent) between channels 1 and 2, which are obtained at each singular variation interval (in percent) considered in the frequency diagram of Fig. 17, are presented in Fig. 18. 
Observing Fig. 18, the amplitude variations over each interval in the number of samples are in the range $[-1.9 \% ; 0.9 \%]$ and, thus, represent very low variations. An SD of $0.45 \%$, a mode of $-0.50 \%$, and an absolute mean of $0.22 \%$ was obtained.

\section{CONCLUSion AND Future Work}

From the high level of statistical similarity between the signals obtained for orthogonal projection directions, we can conclude that acquiring data along a single projection direction is sufficient to correctly characterize the hairiness of a yarn fiber, that is, the direction of hairs protruding from the yarn tested appears to be sufficiently random, yielding almost identical results in both orthogonal projections. Furthermore, the single projection data can simply be corrected by a factor of $\pi / 2$ to obtain a reliable overall characterization of hairiness for the yarn, provided a sufficient number of samples are acquired.

The developed setup has proven to be an accurate method to measure yarn hairiness using only a single light source direction, which reduces the cost and complexity of the final apparatus.

Further work will include the design of the module of image processing for the automatic detection of yarn production characteristics, including the twist step of folded yarns, the fiber twist and the yarn twist orientation, and the number of cables [folded yarn (more than one cable) and nonfolded yarn (only one cable)].

\section{REFERENCES}

[1] V. Carvalho, P. Cardoso, M. Belsley, R. Vasconcelos, and F. Soares, "Determination of yarn hairiness using optical sensors," in Proc. EUROSENSORS XX, Gothenburg, Sweden, Sep. 17-19, 2006.

[2] V. Carvalho, P. Cardoso, M. Belsley, R. Vasconcelos, and F. Soares, "Optical quantification of yarn hairiness using projections along a single direction," in Proc. 9th IASTED Control Appl., Montreal, QC, Canada, May 30-Jun. 1, 2007.

[3] V. Carvalho, P. Cardoso, M. Belsley, R. Vasconcelos, and F. Soares, "Development of a yarn evenness measurement and hairiness analysis system," in Proc. IECON, Paris, France, Nov. 7-10, 2006, pp. 3621-3626.

[4] V. Carvalho, P. Cardoso, M. Belsley, R. Vasconcelos, and F. Soares, "Yarn hairiness parameterization using a coherent signal processing technique," Sens. Actuators A, Phys., vol. 142, no. 1, pp. 217-224, Mar. 2008.

[5] V. Carvalho, P. Cardoso, M. Belsley, R. Vasconcelos, and F. Soares, "A new statistical reference method for yarn hairiness quantification," in Proc. ISIE, Vigo, Spain, Jun. 4-7, 2007, pp. 1864-1869.

[6] V. Carvalho, P. Cardoso, M. Belsley, R. Vasconcelos, and F. Soares, "Optical yarn hairiness measurement system," in Proc. INDIN, Vienna, Austria, Jul. 23-27, 2007, pp. 359-364.

[7] [Online]. Available: http://business.vsnl.com/balasubramanian/hairiness. html

[8] M. Kuzanski, "Measurement methods for yarn hairiness analysisThe idea and construction of research standing," in Proc. 2nd Int. Conf. Young Scientists (MEMSTECH), Lviv, Ukraine, May 24-27, 2006, pp. $87-90$.

[9] J. W. Goodman, Introduction to Fourier Optics. Greenwood Village, CO: McGraw-Hill, 1996.

[10] E. G. Steward, Fourier Optics: An Introduction. New York: Dover, 2004.

[11] P. M. Duffieux, The Fourier Transform and Its Applications to Optics, 2nd ed. New York: Wiley, 1983.

[12] C. K. Madsen, Optical Filter Design and Analysis: A Signal Processing Approach. New York: Wiley-Interscience, 1999.

[13] S. Franco, Design With Operational Amplifiers and Analogue Integrated Circuits, 3rd ed. Greenwood Village, CO: McGraw-Hill, 2001.

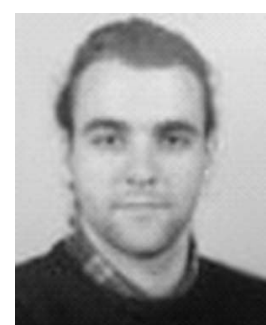

Vítor H. Carvalho received the Licenciatura degree in industrial electronics engineering in the area of telecommunications and industrial informatics, the M.Sc. degree in industrial electronics in the area of automation and robotics, and the Ph.D. degree in industrial electronics, which covers the subject presented in this paper, from Minho University, Guimarães, Portugal, in 2002, 2004, and 2008, respectively.

Since 2005, he has been a Lecturer with the Polytechnic Institute of Cávado and Ave (IPCA), Barcelos, Portugal, and the Catholic University of Portugal (UCP), Porto, Portugal. His main fields of interest are industrial informatics, data acquisition, and signal processing.

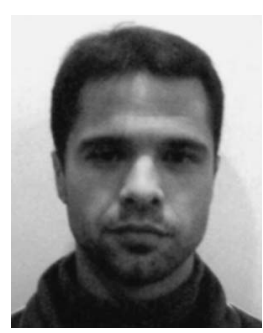

Paulo J. Cardoso received the Licenciatura degree in applied physics from Porto University, Porto, Portugal, in 2002. He is currently working towards the Ph.D. degree in physics with the Department of Physics (DF), Minho University, Braga, Portugal.

His main fields of interest are optical signal processing and nonlinear optics.

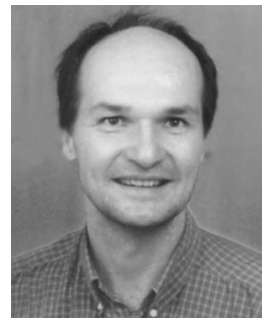

Michael S. Belsley received the Ph.D. degree in physics from the University of Colorado at Boulder in 1986.

$\mathrm{He}$ has been with the California State University at Long Beach; Oxford University, Oxford, U.K; and the University of Oregon, Eugene. He is currently with Minho University, Braga, Portugal, where he has been an Associate Professor of physics since 1992. His main fields of interest are laser spectroscopy and nonlinear optics.

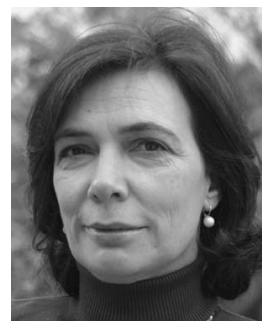

Rosa M. Vasconcelos received the Licenciatura degree in textile engineering and the Ph.D. degree in engineering - textile technology and chemistry, specializing in textile technology, from Minho University, Guimarães, Portugal, in 1984 and 1993, respectively.

Since 2005, she has been an Associate Professor with the Department of Textile Engineering (DET), Minho University. Her fields of interest are textile processes and industrial automation.

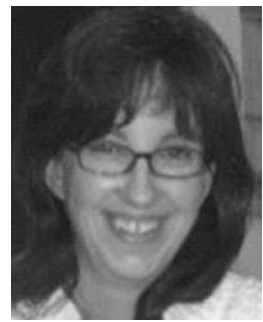

Filomena O. Soares received the Licenciatura and $\mathrm{Ph} . \mathrm{D}$. degrees in chemical engineering from Porto University, Porto, Portugal, in 1986 and 1997, respectively.

In 1997, she joined the Department of Industrial Electronics, Minho University, Guimarães, Portugal, where she is currently an Associate Professor. Her fields of interest are process modeling and control and process automation. 